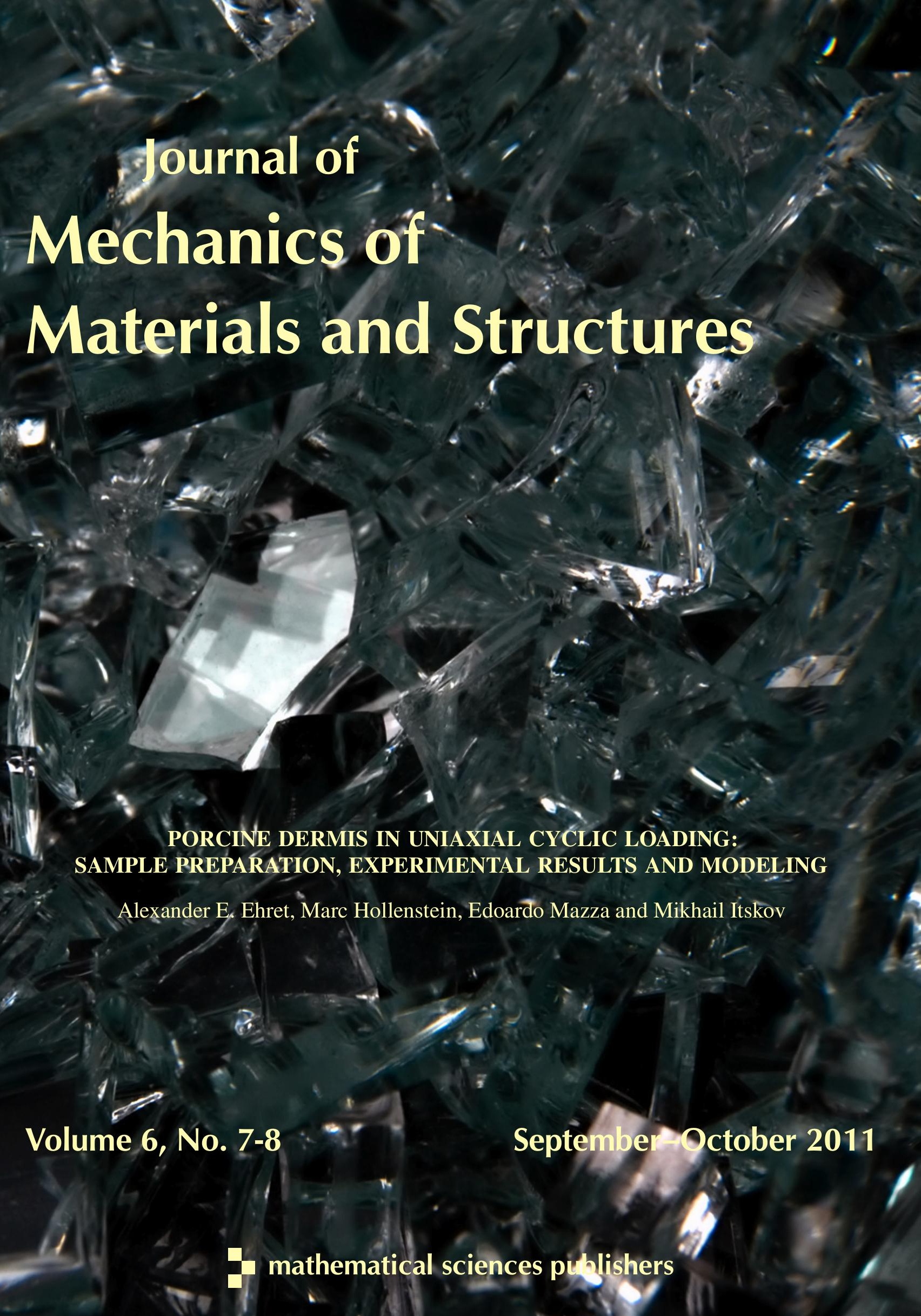




\title{
PORCINE DERMIS IN UNIAXIAL CYCLIC LOADING: SAMPLE PREPARATION, EXPERIMENTAL RESULTS AND MODELING
}

\begin{abstract}
Alexander E. Ehret, Marc Hollenstein, Edoardo Mazza And Mikhail Itskov
We present a straightforward technique to prepare thin samples of planar or bulky soft tissue with very accurate geometry. The experimental procedure includes the preparation of thin slices of tissue by means of a surgical dermatome and specimen extraction by die cutters. We illustrate this method in application to porcine dermal tissue. The prepared specimens were subjected to uniaxial cyclic tension along and across the lines of cleavage with increasing upper stretch limits. Besides a distinct anisotropic and nonlinear behavior, cyclic loading caused considerable preconditioning effects including softening and substantial residual deformations. This observed behavior is well represented by a recently proposed constitutive model accounting for the elastic and dissipative behavior of soft tissues.
\end{abstract}

\section{Introduction}

Skin provides the barrier of the body to the surrounding environment and is for this reason, more directly than any other organ, subject to external loads, possibly going beyond the physiological level. A comprehensive understanding of the mechanical properties of skin is thus of major importance. Moreover, skin is widely used as an autograft in reconstructive surgery, for example, for treating burn wounds. In this field, as well as in plastic surgery, techniques and results can be improved taking the mechanical characteristics of the skin into account. These properties may also serve as an indicator for connective tissue disorders which alter the constitution of the skin. Like the majority of soft biological tissues, skin is a composite material containing a large amount of fibrous proteins, in particular collagen, which, due to a preferred alignment, induce anisotropic material properties. This anisotropy was already discovered in the early 19th century when Dupuytren [1834] investigated the apparent discrepancy between the circular cross-section of a weapon injuring the skin and the resulting elliptic shape of the wound. The preferred directions of this anisotropy coincide with the lines of cleavage of the skin referred to as Langer's lines [Langer 1861].

For the mechanical characterization of skin, a number of experiments have been proposed using in vivo methods such as indentation [Delalleau et al. 2006] and suction [Alexander and Cook 1977], as well as dedicated extension [Alexander and Cook 1977; Khatyr et al. 2004]. The most common in vitro experiment is the uniaxial tension test. Ridge and Wright [1966] carried out uniaxial tensile experiments on human skin specimens and confirmed the anisotropy of skin by a stress response, which was remarkably stiffer along than across the Langer's lines. Further uniaxial tension tests were performed, for example, on the skin of cats [Veronda and Westmann 1970], rats [Haut 1989; Eshel and Lanir 2001;

The advice and support of Prof. Dr. med. A. Prescher, University Hospital Aachen, and Dr. med. M. Guggenheim, University Hospital Zurich, concerning the histology of skin and the proper use of the surgical dermatome is gratefully acknowledged. For technical assistance with the parameter identification process, we thank A. R. Elliott. The work of MH and EM was partially supported by the Swiss National Science Foundation (NCCR CO-ME).

Keywords: dermis, anisotropy, constitutive modeling, preconditioning, soft tissues, mechanical testing, cyclic loading. 
Delalleau et al. 2006], and mice [Del Prete et al. 2004; Muñoz et al. 2008]. Since physiologically relevant loading states are of a biaxial nature, Lanir and Fung [1974] investigated the biaxial viscoelastic stress response of rectangular rabbit skin samples, the width of which was fixed during elongation. By means of a multiaxial testing device, the planar orthotropic elastic properties of human skin were studied in [Reihsner et al. 1995], which confirmed only small deviations of the principal stress directions from the Langer's lines. For an overview of available testing methods, the reader is also referred to the review articles [Payne 1991; Edwards and Marks 1995; Xu et al. 2008].

While the majority of investigations focus on elastic and viscoelastic properties as well as on failure, the anisotropic inelastic softening and, in particular, the preconditioning behavior of skin occurring during the initial few applied load cycles have rarely been the scientific focus. In this regard, Muñoz et al. [2008] have presented an interesting study on the inelastic behaviour of murine skin subject to uniaxial cyclic loading, where the load was successively increased in each step. Besides remarkable residual strains growing with the achieved maximal stretch, they found softening characteristics which clearly resemble the Mullins effect [Mullins 1947] in rubber-like materials. The anisotropic preconditioning behavior of thin porcine dermis samples subject to pure shear loading has recently been investigated in [Hollenstein et al. 2011] and this study will be extended to the case of uniaxial tension in the present work.

After preconditioning, the response of skin is characterized by a stable hysteresis loop [Tong and Fung 1976]. Accordingly, hyperelastic and viscoelastic models dominate the constitutive modeling approaches, including both phenomenological and microstructural strategies; see [Xu et al. 2008] for a review. As for most other tissues, the transient softening behavior during initial load cycles is often treated as a side effect and is only addressed in few works. For example, preconditioning has been modeled for tendon [Sverdlik and Lanir 2002], liver [Nava et al. 2004], and ventricular myocardium [Emery et al. 1997]. In all three works, quasilinear viscoelastic models were enriched either by a plastic or a softening variable to capture the observed softening effects. Under unphysiological conditions, the stress softening is mostly related to tissue damage and consequently is modeled in the framework of continuum damage mechanics, as, for example, applied to overstretched arteries [Balzani et al. 2006] or ligaments [Calvo et al. 2007]. Rubin and Bodner [2002] proposed a viscoplastic model for skin and the underlying fascial tissue, which has recently been applied to simulate the aging skin of the human face [Barbarino et al. 2009].

In the present work, we apply a novel method to excise thin specimens of accurate geometry from planar or bulky soft tissues [Hollenstein et al. 2011]. By using a surgical dermatome, $500 \mu \mathrm{m}$-thick samples of porcine dermis were prepared and subjected to cyclic quasistatic uniaxial loading. The observed stress-response is modeled by a recently proposed modeling framework to capture preconditioning and softening effects [Ehret and Itskov 2009].

\section{Materials and methods}

The preparation of the specimens was carried out according to a protocol recently presented in conjunction with a pure shear test series of porcine dermal tissue and is briefly summarized in this section. For a detailed description and technical specifications please refer to [Hollenstein et al. 2011]. All experiments were performed in accordance with Swiss federal ethical research standards.

2A. Sample preparation. Porcine skin is anatomically subdivided into the epidermal, dermal, and subcutaneous layers, with respective thicknesses of about $30-140 \mu \mathrm{m}, 1-2 \mathrm{~mm}$, and up to $12 \mathrm{~mm}$ or more, 
depending on sex, anatomical site, and nutritional state of the pig [Vardaxis et al. 1997]. Light and scanning electron microscopy [Meyer et al. 1982] as well as confocal laser scanning microscopy [Vardaxis et al. 1997] reveal that porcine dermal tissue is mainly composed of a dense three-dimensional network of collagen fibers and thick fiber bundles crossing each other in two main directions. Ridge and Wright [1966] have suggested a simplified planar lattice structure in which these preferred fiber directions of skin form an angle of "somewhat less than $45^{\circ}$ " to the Langer's lines. The resulting axes of orthotropy along and across the Langer's lines indeed coincide well with experimental results (see, for example, [Reihsner et al. 1995]). Depending on the anatomical site, a substantial amount of elastic fibers are also present [Meyer et al. 1981]. The two proteins collagen and elastin constitute about $70-80 \%$ and $4 \%$ of the dry weight, respectively [Mathews 1975; Fung 1993].

Skin pieces from the snout and head region of female domestic pigs (Sus scrofa domestica) were obtained from the slaughterhouse immediately after animal slaughter. The pieces were approximately $300 \times 300 \mathrm{~mm}$ large with a thickness of about $10-15 \mathrm{~mm}$, containing the subcutaneous layer. After carefully being rinsed with water, the pieces were shaved and finally stored in saline-soaked cloths at $4^{\circ} \mathrm{C}$.

Following the protocol proposed in [Hollenstein et al. 2011], the pieces were first nailed under slight tension on a foam damping pad and paraffin oil was applied to the skin surface. Thereafter $500 \mu \mathrm{m}$-thick and $80 \mathrm{~mm}$-wide slices were extracted by means of a surgical dermatome (Figure 1a). The second and third layers extracted in this way consisted completely of dermal tissue and were used for testing.

Finally, rectangular specimens were punched out of the dermis sections by the use of a $10 \times 80 \mathrm{~mm}$ die cutter (Figure 1b) and shortened to a length of $50 \mathrm{~mm}$ with the aid of a scalpel. To obtain longitudinal and transversal specimens, respectively, the die was aligned with its long side either along or perpendicular to the optically observable crease lines, which coincide well with the cleavage lines [Cox 1941].

2B. Experimental setup and test realization. Uniaxial tension tests were performed on a custom-made testing device with two horizontally arranged hydraulic actuators with an available piston rod stroke of $100 \mathrm{~mm}$. Load cells with a capacity of $100 \mathrm{~N}$ were installed at the end of each actuator. Custom-made

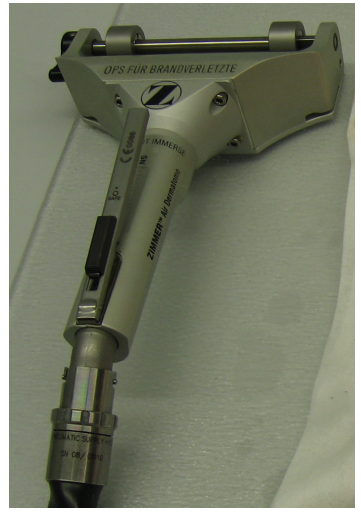

(a)

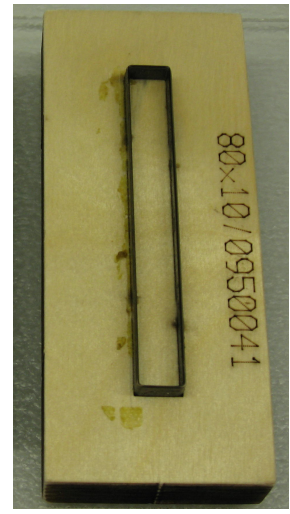

(b)

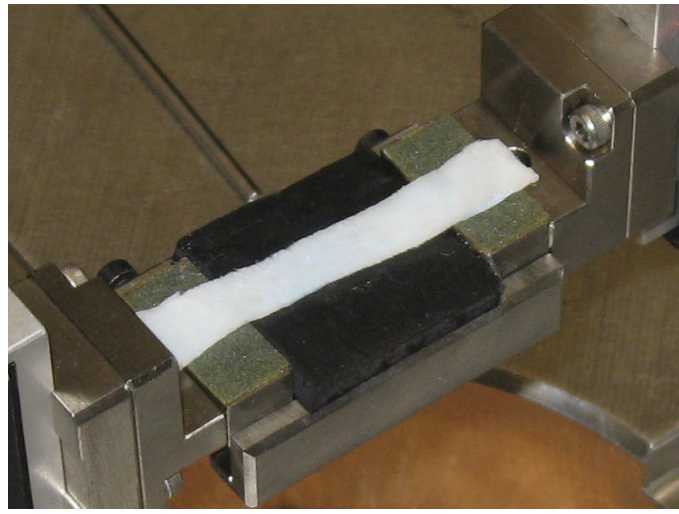

(c)

Figure 1. (a) Surgical air-dermatome. (b) Custom-made die cutter for excision of uniaxial specimens. (c) Dermis specimen on the supporting table before mounting the upper grips. 
titanium clamps equipped with sandpaper allowed for specimen fixation by closing a knurled thumb screw. The force $F$ acting on the fixed actuator and the displacement of the moving actuator relative to its initial position were recorded.

For precise spreading of the specimens on the grip surfaces, a small supporting table was installed in between the clamps while the actuator piston rods were in their initial testing position, providing a distance of $30 \mathrm{~mm}$ (Figure 1c). After the closing of the clamps and removal of the table, the specimens were hanging slightly slack in between the two clamps, and a preload of $0.05 \mathrm{~N}$ was applied to straighten the sample. The resulting length is considered as the reference length. Note that the choice of this preload thus has significant influence on the stretch calculation. Before and during testing, the samples were sprayed with physiological saline in order to keep them moist.

The induced nominal strain $\varepsilon_{\mathrm{n}}(t)$, the stretch ratio $\lambda_{\mathrm{n}}(t)$, and the nominal stress $P$ were computed as [Hollenstein et al. 2011]

$$
\varepsilon_{\mathrm{n}}(t)=\frac{d(t)}{l_{0}}, \quad \lambda_{\mathrm{n}}(t)=1+\varepsilon_{\mathrm{n}}(t), \quad P=\frac{F}{A_{0}},
$$

where $d(t)$ is the displacement of the actuator, $A_{0}$ the initial cross-sectional area, $l_{0}$ the free gauge length at preload, and $F$ the measured force.

We performed the tests in a displacement-controlled mode, where the protocol was defined based on the nominal strain and its rate. The lower cycle-reversal points, however, were enforced when the measured force became less than $0.05 \mathrm{~N}$ in order to prevent the tissue from becoming slack during the cyclic loading. The preconditioning protocol contained five sets of five cycles, respectively, with increasing upper strain levels of $2.9 \%, 5.7 \%, 8.5 \%, 11.4 \%$, and $14.3 \%$. The highest strain level was chosen well below the rupture strain in preceding tension-to-failure experiments and the lower levels were obtained by appropriate scaling. All tests were run with $\dot{\varepsilon}_{\mathrm{n}}=0.1 \% \mathrm{~s}^{-1}$. In previous experiments, we found that further decreasing the rate below this value had negligible influence on the tissue response, which was thus considered quasistatic.

\section{Experimental results}

In Figure 2 the nominal stress $P$ in the loading direction is plotted against the applied stretch ratio for a longitudinal and a transversal specimen cut from the same skin sample. As typical for the majority of soft biological tissues each stress-stretch curve is characterized by a $J$-shaped form with a distinct toe region. Comparison between the two graphs reveals the distinct anisotropy of the tissue, with a much stiffer response for loading along the direction of the cleavage lines.

All specimens showed substantial preconditioning effects. This includes stress softening and a tendency to stabilise after some cycles if the upper stretch limit is kept constant. Increasing this limit, however, the tissue again undergoes several preconditioning cycles until a reasonably stable response is reached. It is remarked that even for loading at these low strain rates, the stabilized loading-unloading cycles demonstrate hysteresis, in line with the phenomenon of pseudoelasticity [Fung 1993]. Along with the softening, both longitudinal and transversal samples accumulate remarkably large residual strains of approximately $60 \%$ of the applied strain after removing the load. The observed phenomena, in particular the strong effect of the previous loading history, bear a tremendous resemblance to stress softening characteristics of rubber-like materials such as the Mullins effect [Mullins 1947]. 

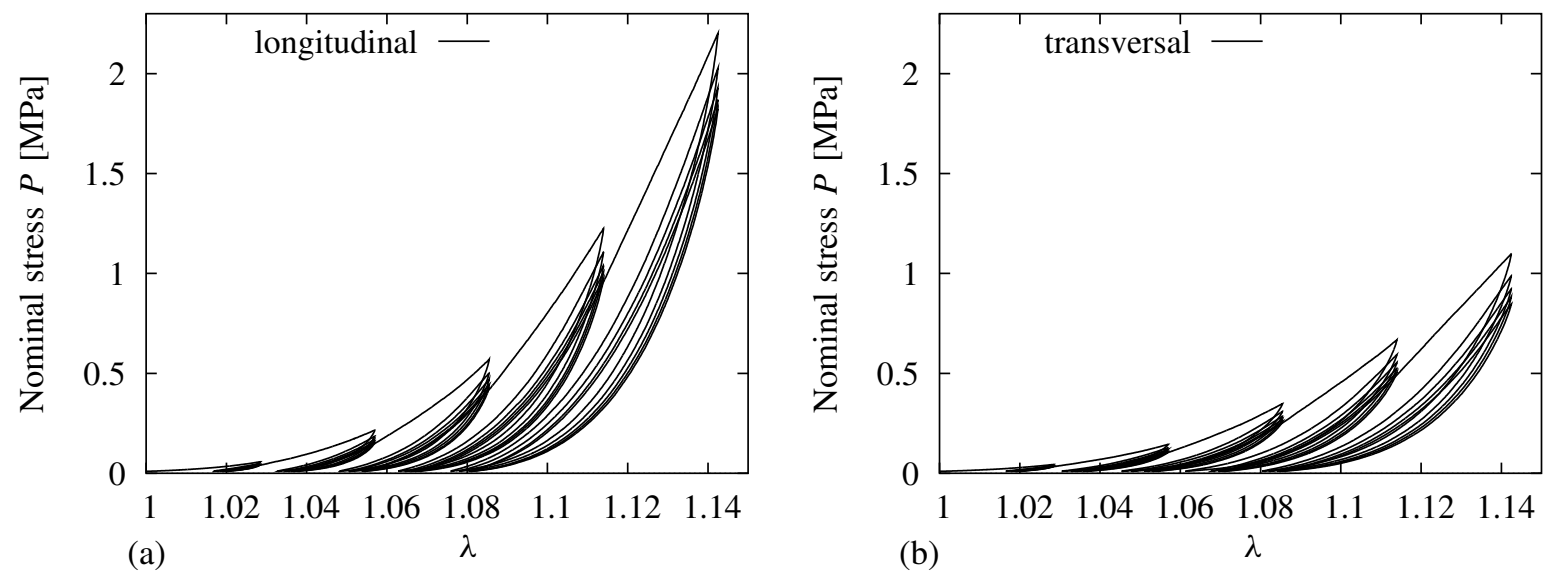

Figure 2. Nominal stress response of two dermis specimens cut either (a) along or (b) perpendicular to the cleavage lines of the skin.

\section{Constitutive modeling}

In order to interpret the observed anisotropic softening behavior, we consider a recently proposed dissipative modeling framework for anisotropic materials [Ehret and Itskov 2009]. The model treats soft biological tissues in the framework of classical invariant theory of fiber-reinforced materials consisting of an isotropic matrix and $n$ families of collagen fibers, the alignment of which is specified by unit vectors $\boldsymbol{a}_{i}, i=1,2, \ldots, n$. Based on these fiber vectors, the structural tensors $\boldsymbol{L}_{i}=\boldsymbol{a}_{i} \otimes \boldsymbol{a}_{i}$ are introduced and, by means of the second-order identity tensor $\boldsymbol{I}$, additionally $\boldsymbol{L}_{0}=\boldsymbol{I} / 3$, which accounts for the isotropic matrix. A set of suitable invariants to formulate the free energy function is given in terms of the structural tensors and the right Cauchy-Green tensor $\boldsymbol{C}$ as (for details, refer to [Ehret and Itskov 2007])

$$
I_{i}=\operatorname{tr}\left(\boldsymbol{C} \boldsymbol{L}_{i}\right), \quad J_{i}=\operatorname{tr}\left[(\operatorname{cof} \boldsymbol{C}) \boldsymbol{L}_{i}\right], \quad \mathrm{III}_{\boldsymbol{C}}=\operatorname{det} \boldsymbol{C}, \quad i=0,1, \ldots, n,
$$

where $\operatorname{cof} \boldsymbol{C}=\boldsymbol{C}^{-1} \operatorname{det} \boldsymbol{C}$. In the case $i=0$, one finds $I_{0}=\mathrm{I}_{\boldsymbol{C}} / 3$ and $J_{0}=\mathrm{II}_{\boldsymbol{C}} / 3$, where $\mathrm{I}_{\boldsymbol{C}}$ and $\mathrm{II}_{\boldsymbol{C}}$ denote the first and second principal invariants of $\boldsymbol{C}$. Moreover, for $i=1,2, \ldots, n, I_{i}$ describes the change in squared length of a line element aligned with the vector $\boldsymbol{a}_{i}$. Using Nanson's formula, one further realises that $J_{i}$ accounts for the change in squared area of an element with the surface normal $\boldsymbol{a}_{i}$ in the reference state [Schröder and Neff 2003]. Under the incompressibility constraint $\operatorname{det} \boldsymbol{C}=1$, the invariants (4-1) take the form

$$
I_{i}=\operatorname{tr}\left(\boldsymbol{C} \boldsymbol{L}_{i}\right), \quad K_{i}=\operatorname{tr}\left[\boldsymbol{C}^{-1} \boldsymbol{L}_{i}\right], \quad \mathrm{III}_{\boldsymbol{C}}=1, \quad i=0,1, \ldots, n,
$$

where the second set of invariants is denoted by $K_{i}$ in this case. Taking into account that the isotropic matrix and various fibers may have different influences on the deformation behavior, generalized invariants are postulated as linear combinations of the form [Ehret and Itskov 2007]

$$
\tilde{I}=\sum_{i=0}^{n} u_{i} I_{i}, \quad \tilde{K}=\sum_{i=0}^{n} v_{i} K_{i}
$$


where $u_{i}$ and $v_{i}$ denote nonnegative weight factors controlling the influence of the different constituents, respectively. As a result of the kinematic interpretation of the invariants (4-2), $u_{i}$ is associated with longitudinal characteristics, while $v_{i}$ relates to cross-sectional properties. The inelastic modeling concept is based on the idea that structural alterations of a constituent $i$ must be reflected in a change of the associated weight factor. Treating the weight factors as internal variables characterising the structural state of the tissue, its free energy $\Psi$ per unit volume is represented in the incompressible case $(\operatorname{det} C=1)$ by [Itskov and Ehret 2009]

$$
\Psi=\tilde{\Psi}(\tilde{I}, \tilde{K})=\hat{\Psi}\left(I_{i}, K_{i}, u_{i}, v_{i}\right)=\frac{\mu}{4}\left\{\frac{1}{\alpha}\left[e^{\alpha(\tilde{I}-1)}-1\right]+\frac{1}{\beta}\left[e^{\beta(\tilde{K}-1)}-1\right]\right\},
$$

where $\mu$ has dimensions of stress and $\alpha>0$ and $\beta>0$ denote dimensionless material constants. Keeping the weight factors constant, the free energy function (4-4) reduces to the incompressible representation of a recently proposed polyconvex and coercive strain-energy function [Ehret and Itskov 2007]. In the elastic case, this property guarantees material stability as well as the existence of the global minimiser of the total elastic energy, which is an important prerequisite for the solution of boundary value problems [Ball 1977; Ciarlet 1988; Schröder and Neff 2003].

Inserting (4-4) into the Clausius-Duhem inequality and regarding the incompressibility constraint yields the second Piola-Kirchhoff stress tensor as $\boldsymbol{S}=2 \partial \Psi / \partial \boldsymbol{C}-p \boldsymbol{C}^{-1}$ where $p$ is an arbitrary scalar. Furthermore, one obtains a dissipation inequality that guarantees a nonnegative rate of entropy production [Truesdell and Noll 1965]. Borrowing methods from strain space plasticity [Naghdi and Trapp 1975], one can set up evolution criteria and suitable rate equations for the weight factors so that the latter inequality is satisfied and the model is thermodynamically consistent. The evolution equations are formulated in terms of the thermodynamic forces ${ }^{n} \varphi_{i}=\partial \Psi / \partial u_{i}$ and ${ }^{v} \varphi_{i}=\partial \Psi / \partial v_{i}$, work-conjugate to $u_{i}$ and $v_{i}$, respectively. In the special case of a continuously softening material, these evolution equations take the form [Ehret and Itskov 2009]

$$
\dot{u}_{i}=\left\{\begin{array}{ll}
{ }^{u} d_{i}{ }^{u} \stackrel{\circ}{\varphi}_{i} & \text { if }{ }^{u} \stackrel{\circ}{\varphi}_{i}>0 \wedge I_{i}>1, \\
0 & \text { else, }
\end{array} \dot{v}_{i}= \begin{cases}{ }^{v} d_{i}{ }^{v} \stackrel{\circ}{\varphi}_{i} & \text { if }{ }^{v} \stackrel{\circ}{\varphi}_{i}>0 \wedge K_{i}>1, \\
0 & \text { else, }\end{cases}\right.
$$

for $i=0,1, \ldots, n$, where the superposed circle indicates $\stackrel{\circ}{x}=\partial x / \partial \boldsymbol{C}: \dot{\boldsymbol{C}}$, and ${ }^{u} d_{i} \leq 0$ and ${ }^{v} d_{i} \leq 0$ denote softening functions.

A structural initial state is introduced such that the tissue is energy and stress-free in the reference configuration with $\boldsymbol{C}=\boldsymbol{I}$. Considering the latter relation in (4-4) and the resulting second Piola-Kirchhoff stress tensor, one easily confirms that the tissue is energy and stress-free if [Itskov and Ehret 2009]

$$
u_{i}=v_{i}=w_{i}, \quad \sum_{i=0}^{n} w_{i}=1,
$$

which will be referred to as the virgin state of the material.

\section{Comparison with experimental data}

5A. Model specification. Taking the initially discussed arrangement of collagen fibers in the dermis into account, we adopt the simplified fiber structure suggested in [Ridge and Wright 1966] and model the tissue as a fiber-reinforced material with two fiber families forming an angle of $\pm \theta$ with the cleavage 
lines. Introducing the vectors $\boldsymbol{e}_{1}$ along and $\boldsymbol{e}_{2}$ across these lines, as well as $\boldsymbol{e}_{3}$ in the thickness direction, they form an orthonormal basis with respect to which the two fiber vectors are specified as

$$
\boldsymbol{a}_{1}=\cos (\theta) \boldsymbol{e}_{1}+\sin (\theta) \boldsymbol{e}_{2}, \quad \boldsymbol{a}_{2}=\cos (\theta) \boldsymbol{e}_{1}-\sin (\theta) \boldsymbol{e}_{2} .
$$

For loading along and across the fiber directions, the nominal stresses can thus be calculated in terms of the principal stretches $\lambda_{k}, k=1,2,3$, by

$$
P_{k}=\frac{\partial \Psi}{\partial \lambda_{k}}-p \lambda_{k}^{-1},
$$

where $p$ denotes an arbitrary parameter which is determined by the assumption of stress-free lateral boundaries during the uniaxial tests. As a result of the incompressibility condition, the principal stretches are related by $\lambda_{3}=1 /\left(\lambda_{1} \lambda_{2}\right)$, so that the nominal stresses may be expressed in the form $P_{k}=\bar{P}_{k}\left(\lambda_{1}, \lambda_{2}\right)$.

We assign the observed softening exclusively to alterations of the longitudinal fiber properties; the bulk and cross-sectional properties remain unchanged so that $u_{0}=v_{0}=w_{0}$ and $v_{i}=w_{i}, i=1,2$. Thus it remains to specify the softening functions in the evolution equations (4-5) 1 for $u_{i}, i=1,2$, where with minor changes the form proposed in [Ehret and Itskov 2009] is adopted as

$$
{ }^{u} d_{i}=\frac{4^{u} k_{i}}{\mu}\left(\bar{u}_{i}-u_{i}\right), \quad \bar{u}_{i}=w_{i} \exp \left[-{ }^{u} c_{i}\left(\frac{{ }^{u} \bar{\varphi}_{i}}{\mu / 4}\right)^{u} b_{i}\right], \quad{ }^{u} \bar{\varphi}_{i}=\max _{\tau \in(-\infty, t]}\left[{ }^{u} \varphi_{i}(\tau)\right],
$$

where ${ }^{u} \bar{\varphi}_{i}$ indicates the maximum of the thermodynamic force reached in the past loading history, and ${ }^{u} k_{i},{ }^{u} b_{i}$, and ${ }^{{ }^{u} c_{i}}$ are dimensionless constants. In the virgin state, the two fiber families are assumed to be mechanically equivalent, and thus $w_{1}=w_{2}=\left(1-w_{0}\right) / 2$, in view of (4-6). This equivalence also motivates the evolution of these factors being governed by analogous evolution equations so that ${ }^{u} k_{i}$, ${ }^{u} b_{i}$, and ${ }^{u} c_{i}$ take the same value for $i=1$ and $i=2$, respectively. Thus altogether there are three constants characterising the anisotropic softening as well as the parameters $\mu, \alpha, \beta$, and $w_{0}$ and the angle $\theta$ controlling the elastic response.

5B. Parameter identification. In order to determine the aforementioned parameters in comparison with the uniaxial tension tests, the constitutive equations were implemented in a Maple 10 worksheet. By this means, the model response was simulated for the applied stretch history in the loading direction. For each stretch increment, the evolution equations were solved implicitly and the stretch in the lateral direction was computed from the condition that this direction be stress-free. With these values at hand, the stress in loading direction was calculated. The model does, however, account only for the dissipative processes, inducing softening, and not for the hysteresis loops formed by unloading and subsequent reloading curves. For this reason, the difference between the model and experiment was only taken into account for the 25 unloading paths, where $N_{l}^{k}$ data points for longitudinal and $N_{t}^{k}$ data points for transversal loading were considered on the $k$-th unloading curve. Based on these differences, a least-squares objective function of the general form

$$
\Omega=\sum_{k=1}^{25}\left\{\frac{\omega_{l}^{k}}{N_{l}^{k}} \sum_{i=1}^{N_{l}^{k}}\left[\bar{P}_{1}\left(\lambda_{l}^{k, i}, \lambda_{2}^{k, i}\right)-P_{l}^{k, i}\right]^{2}+\frac{\omega_{t}^{k}}{N_{t}^{k}} \sum_{i=1}^{N_{t}^{k}}\left[\bar{P}_{2}\left(\lambda_{1}^{k, i}, \lambda_{t}^{k, i}\right)-P_{t}^{k, i}\right]^{2}\right\}
$$

was defined and subjected to an optimization procedure. Here $\left(\lambda_{l}^{k, i}, P_{l}^{k, i}\right)$ and $\left(\lambda_{t}^{k, i}, P_{t}^{k, i}\right)$ are experimental data pairs, $\lambda_{1,2}^{k, i}$ denote the computed lateral stretches, and $\omega_{l, t}^{k}$ are weighting factors. 

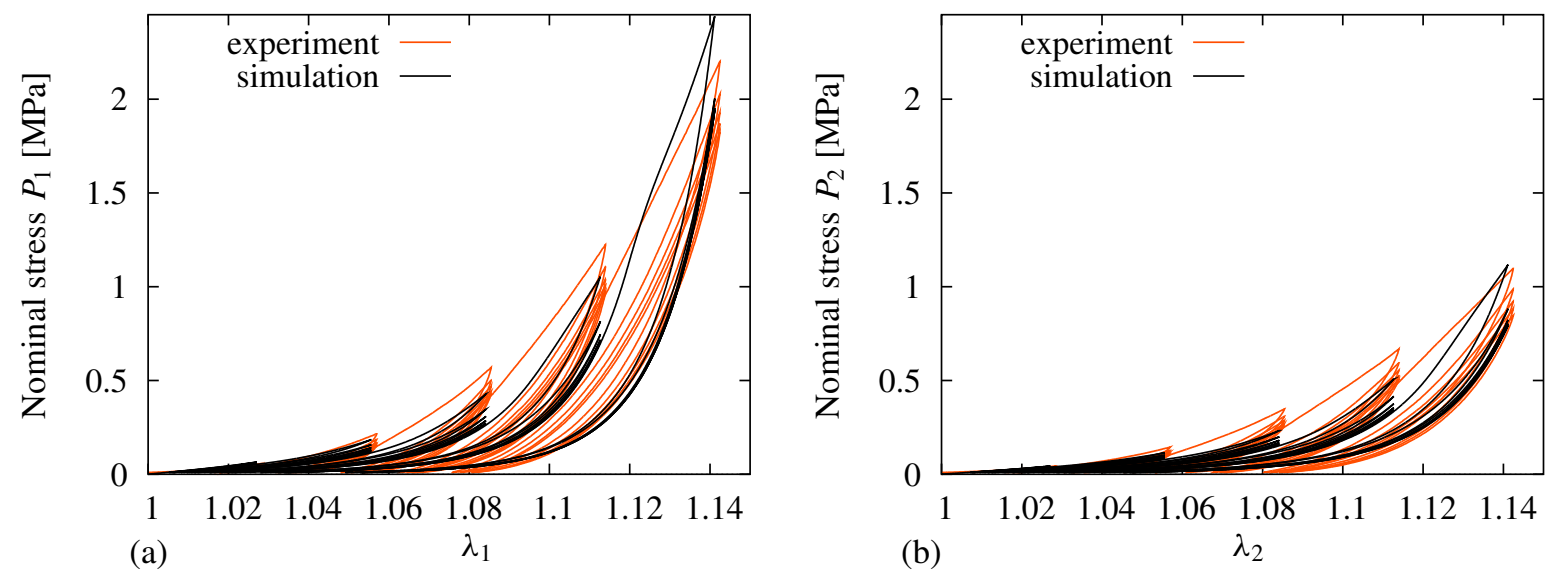

Figure 3. Comparison of the simulated stress response based on the parameters obtained by the fitting procedure with experimental data for loading (a) along and (b) across the lines of cleavage. Material parameters: $\mu=0.9545 \mathrm{MPa}, \alpha=146.2, \beta=170.0$, $w_{0}=0.2348, \theta=42.44^{\circ},{ }^{u} k_{1}=0.1420,{ }^{u} b_{1}=0.4453$, and ${ }^{u} c_{1}=0.01306$.

Based on the parameter values obtained through the fitting procedure, we simulated the model stress response to the protocol applied in the experiments. The simulation results displayed in Figure 3 (the black solid lines) show fair agreement with the experimental stresses, where we emphasise once again that the hysteresis loops formed by the successive reloading paths are not taken into account by the model. Note that, although the angle between fiber bundles and Langer's lines was estimated at close to $\theta=45^{\circ}$, the responses for loading along and across these lines differ remarkably, which is explained by the strong nonlinearity of the model.

\section{Conclusions}

In the present paper, we used a novel and robust protocol for the preparation of uniaxial tension specimens of accurately defined dimensions. Sample preparation from soft biological tissues is challenging: shape and structure of the available sample material limit possible geometries and thus applicable extraction procedures. During cutting, the tissue is locally disrupted and new boundaries are created. This may lead to artefactual behavior which affects the results of the mechanical characterization. Thus, gently prepared samples with exact geometry are highly valuable for in vitro testing.

The key features of the preparation protocol include the use of a surgical dermatome in combination with punching dies. The dermatome allows for obtaining thin and thus relatively homogeneous tissue sections. The punching tools guarantee a precise planar geometry of the specimens. Dermatomes are designed to perform extremely smooth sectioning in plastic and reconstructive surgery, and are thus highly suitable for the preparation of evenly shaped specimens for materials testing. Therefore, we argue that, due to the precise geometry of the samples obtained, the proposed method of sample preparation leads to a general reduction in systematic errors, which will have to be proven statistically on a broader database in the future.

Concerning components and structure, dermal tissue is representative of many soft biological tissues and exhibits typical characteristics such as pronounced preconditioning and a response differing in 
loading and unloading, a phenomenon known as pseudoelasticity. The remarkable residual deformations demonstrated by the dermis samples in the present study are in line with recent observations on skin specimens including all layers [Muñoz et al. 2008].

The observed inelastic behavior was taken into account by a constitutive model that regards softening as structural alterations of the longitudinal and cross-sectional fiber properties. The model in its present form accounts only for the softening including residual deformations, not, however, for the hysteresis formed due to pseudoelastic behavior of the tissue during reloading. Consequently, the model was calibrated only against the unloading part of the stress-strain cycles, while neglecting the stiffening during reloading. Bearing this in mind, the obtained agreement between simulation and experiment is remarkable and reveals the potential of the model for future developments.

\section{References}

[Alexander and Cook 1977] H. Alexander and T. H. Cook, "Accounting for natural tension in the mechanical testing of human skin”, J. Investig. Dermatol. 69 (1977), 310-314.

[Ball 1977] J. M. Ball, "Convexity conditions and existence theorems in nonlinear elasticity", Arch. Ration. Mech. An. 63:4 (1977), 337-403.

[Balzani et al. 2006] D. Balzani, J. Schröder, and D. Gross, "Simulation of discontinuous damage incorporating residual stresses in circumferentially overstretched atherosclerotic arteries", Acta Biomater. 2:6 (2006), 609-618.

[Barbarino et al. 2009] G. G. Barbarino, M. Jabareen, J. Trzewik, A. Nkengne, G. Stamatas, and E. Mazza, "Development and validation of a three-dimensional finite element model of the face", J. Biomech. Eng. (ASME) 131:4 (2009), 041006.

[Calvo et al. 2007] B. Calvo, E. Peña, M. A. Martinez, and M. Doblaré, "An uncoupled directional damage model for fibred biological soft tissues: formulation and computational aspects", Int. J. Numer. Methods Eng. 69:10 (2007), 2036-2057.

[Ciarlet 1988] P. G. Ciarlet, Mathematical elasticity, I: Three-dimensional elasticity, 2nd ed., Studies in Mathematics and its Applications 20, North-Holland, Amsterdam, 1988.

[Cox 1941] H. T. Cox, “The cleavage lines of the skin”, Br. J. Surg. 29:114 (1941), 234-240.

[Del Prete et al. 2004] Z. Del Prete, S. Antoniucci, A. H. Hoffman, and P. Grigg, "Viscoelastic properties of skin in Mov-13 and Tsk mice", J. Biomech. 37:10 (2004), 1491-1497.

[Delalleau et al. 2006] A. Delalleau, G. Josse, J.-M. Lagarde, H. Zahouani, and J.-M. Bergheau, "Characterization of the mechanical properties of skin by inverse analysis combined with the indentation test", J. Biomech. 39:9 (2006), 1603-1610.

[Dupuytren 1834] G. Dupuytren, Traité théorique et pratique des blessures par armes de guerre, J.-B. Baillière, Paris, 1834.

[Edwards and Marks 1995] C. Edwards and R. Marks, "Evaluation of biomechanical properties of human skin", Clin. Dermatol. 13:4 (1995), 375-380.

[Ehret and Itskov 2007] A. E. Ehret and M. Itskov, "A polyconvex hyperelastic model for fiber-reinforced materials in application to soft tissues", J. Mater. Sci. 42:21 (2007), 8853-8863.

[Ehret and Itskov 2009] A. E. Ehret and M. Itskov, "Modeling of anisotropic softening phenomena: application to soft biological tissues", Int. J. Plast. 25:5 (2009), 901-919.

[Emery et al. 1997] J. L. Emery, J. H. Omens, and A. D. McCulloch, "Strain softening in rat left ventricular myocardium", J. Biomech. Eng. (ASME) 119:1 (1997), 6-12.

[Eshel and Lanir 2001] H. Eshel and Y. Lanir, "Effects of strain level and proteoglycan depletion on preconditioning and viscoelastic responses of rat dorsal skin", Ann. Biomed. Eng. 29:2 (2001), 164-172.

[Fung 1993] Y. C. Fung, Biomechanics: mechanical properties of living tissues, 2nd ed., Springer, New York, 1993.

[Haut 1989] R. C. Haut, "The effects of orientation and location on the strength of dorsal rat skin in high and low speed tensile failure experiments", J. Biomech. Eng. (ASME) 111:2 (1989), 136-140.

[Hollenstein et al. 2011] M. Hollenstein, A. E. Ehret, M. Itskov, and E. Mazza, "A novel experimental procedure based on pure shear testing of dermatome-cut samples applied to porcine skin", Biomech. Model. Mechanobiol. 10:5 (2011), 651-661. 
[Itskov and Ehret 2009] M. Itskov and A. E. Ehret, "A universal model for the elastic, inelastic and active behaviour of soft biological tissues”, GAMM-Mitt. 32:2 (2009), 221-236.

[Khatyr et al. 2004] F. Khatyr, C. Imberdis, P. Vescovo, D. Varchon, and J.-M. Lagarde, "Model of the viscoelastic behaviour of skin in vivo and study of anisotropy", Skin Res. Technol. 10:2 (2004), 96-103.

[Langer 1861] K. Langer, "Zur Anatomie und Physiologie der Haut, I: Über die Spaltbarkeit der Cutis”, Sitzungsber. Kais. Akad. Wiss. Math.-Naturwiss. Kl. 44 (1861), 19-46. In German; translated in "On the anatomy and physiology of the skin, I: The cleavability of the cutis", Br. J. Plast. Surg. 31:1 (1978), 3-8.

[Lanir and Fung 1974] Y. Lanir and Y. C. Fung, "Two-dimensional mechanical properties of rabbit skin, II: Experimental results", J. Biomech. 7:2 (1974), 171-182.

[Mathews 1975] M. B. Mathews, Connective tissue: macromolecular structure and evolution, edited by A. Kleinzeller et al., Molecular Biology, Biochemistry and Biophysics 19, Springer, Berlin, 1975.

[Meyer et al. 1981] W. Meyer, K. Neurand, and B. Radke, "Elastic fibre arrangement in the skin of the pig", Arch. Dermatol. Res. 270:4 (1981), 391-401.

[Meyer et al. 1982] W. Meyer, K. Neurand, and B. Radke, "Collagen fibre arrangement in the skin of the pig", J. Anat. 134:1 (1982), 139-148.

[Muñoz et al. 2008] M. J. Muñoz, J. A. Bea, J. F. Rodríguez, I. Ochoa, J. Grasa, A. Pérez del Palomar, P. Zaragoza, R. Osta, and M. Doblaré, "An experimental study of the mouse skin behaviour: damage and inelastic aspects", J. Biomech. 41:1 (2008), 93-99.

[Mullins 1947] L. Mullins, "Effect of stretching on the properties of rubber", J. Rubber Res. 16 (1947), 275-289.

[Naghdi and Trapp 1975] P. M. Naghdi and J. A. Trapp, "The significance of formulating plasticity theory with reference to loading surfaces in strain space", Int. J. Eng. Sci. 13:9-10 (1975), 785-797.

[Nava et al. 2004] A. Nava, E. Mazza, O. Haefner, and M. Bajka, "Experimental observation and modelling of preconditioning in soft biological tissues", Lect. Notes Comput. Sci. 3078 (2004), 1-9.

[Payne 1991] P. A. Payne, "Measurement of properties and function of skin”, Clin. Phys. Physiol. Meas. 12:2 (1991), 105-129.

[Reihsner et al. 1995] R. Reihsner, B. Balogh, and E. J. Menzel, "Two-dimensional elastic properties of human skin in terms of an incremental model at the in vivo configuration", Med. Eng. Phys. 17:4 (1995), 304-313.

[Ridge and Wright 1966] M. D. Ridge and V. Wright, "The directional effects of skin: a bio-engineering study of skin with particular reference to Langer's lines”, J. Investig. Dermatol. 46:4 (1966), 341-346.

[Rubin and Bodner 2002] M. B. Rubin and S. R. Bodner, "A three-dimensional nonlinear model for dissipative response of soft tissue", Int. J. Solids Struct. 39:19 (2002), 5081-5099.

[Schröder and Neff 2003] J. Schröder and P. Neff, "Invariant formulation of hyperelastic transverse isotropy based on polyconvex free energy functions", Int. J. Solids Struct. 40:2 (2003), 401-445.

[Sverdlik and Lanir 2002] A. Sverdlik and Y. Lanir, "Time-dependent mechanical behavior of sheep digital tendons, including the effects of preconditioning", J. Biomech. Eng. (ASME) 124:1 (2002), 78-84.

[Tong and Fung 1976] P. Tong and Y.-C. Fung, "The stress-strain relationship for the skin”, J. Biomech. 9:10 (1976), 649-657.

[Truesdell and Noll 1965] C. Truesdell and W. Noll, Die nicht-linearen Feldtheorien der Mechanik, edited by S. Flügge, Handbuch der Physik III/3, Springer, Berlin, 1965.

[Vardaxis et al. 1997] N. J. Vardaxis, T. A. Brans, M. E. Boon, R. W. Kreis, and L. M. Marres, "Confocal laser scanning microscopy of porcine skin: implications for human wound healing studies", J. Anat. 190:4 (1997), 601-611.

[Veronda and Westmann 1970] D. R. Veronda and R. A. Westmann, "Mechanical characterization of skin: finite deformations", J. Biomech. 3:1 (1970), 111-124.

[Xu et al. 2008] F. Xu, T. J. Lu, and K. A. Seffen, "Biothermomechanical behavior of skin tissue", Acta Mech. Sinica 24:1 (2008), 1-23.

Received 1 Jul 2010. Revised 10 Dec 2010. Accepted 10 Jan 2011.

ALEXANDER E. EHRET: ehret@km.rwth-aachen.de 
Department of Continuum Mechanics, RWTH Aachen University, 52056 Aachen, Germany MARC Hollenstein: mhollenstein@ethz.ch Institute for Mechanical Systems, ETH Zurich, 8092 Zurich, Switzerland EDOARDO MAZZA: emazza@ethz.ch Institute for Mechanical Systems, ETH Zurich, 8092 Zurich, Switzerland and

EMPA, 8600 Dübendorf, Switzerland

MiKHAIL ITSKOV: itskov@km.rwth-aachen.de

Department of Continuum Mechanics, RWTH Aachen University, 52056 Aachen, Germany 


\title{
JOURNAL OF MECHANICS OF MATERIALS AND STRUCTURES
}

\author{
jomms.org
}

Founded by Charles R. Steele and Marie-Louise Steele

EDITORS

Charles R. SteEle

DAVIDE BIGONI

Stanford University, USA

YASUHIDE SHINDO

University of Illinois at Urbana-Champaign, USA

Tohoku University, Japan

\section{EDITORIAL BOARD}

$\begin{aligned} \text { H. D. BUI } & \text { École Polytechnique, France } \\ \text { J. P. CARTER } & \text { University of Sydney, Australia } \\ \text { R. M. CHRISTENSEN } & \text { Stanford University, USA } \\ \text { G. M. L. GLADWELL } & \text { University of Waterloo, Canada } \\ \text { D. H. HODGES } & \text { Georgia Institute of Technology, USA } \\ \text { J. HUTCHINSON } & \text { Harvard University, USA } \\ \text { C. HWU } & \text { National Cheng Kung University, Taiwan } \\ \text { B. L. KARIHALOO } & \text { University of Wales, UK } \\ \text { Y. Y. KIM } & \text { Seoul National University, Republic of Korea } \\ \text { Z. MROZ } & \text { Academy of Science, Poland } \\ \text { D. PAMPLONA } & \text { Universidade Católica do Rio de Janeiro, Brazil } \\ \text { M. B. RUBIN } & \text { Technion, Haifa, Israel } \\ \text { A. N. SHUPIKOV } & \text { Ukrainian Academy of Sciences, Ukraine } \\ \text { T. TARNAI } & \text { University Budapest, Hungary } \\ \text { F. Y. M. WAN } & \text { University of California, Irvine, USA } \\ \text { P. WRIGGERS } & \text { Universität Hannover, Germany } \\ \text { W. YANG } & \text { Tsinghua University, China } \\ \text { F. ZIEGLER } & \text { Technische Universität Wien, Austria } \\ & \\ \text { PRODUCTION } & \text { contact@ msp.org } \\ \text { SILVIO LEVY } & \text { Scientific Editor }\end{aligned}$

Cover design: Alex Scorpan

Cover photo: Mando Gomez, www.mandolux.com

See http://jomms.org for submission guidelines.

JoMMS (ISSN 1559-3959) is published in 10 issues a year. The subscription price for 2011 is US \$520/year for the electronic version, and \$690/year (+\$60 shipping outside the US) for print and electronic. Subscriptions, requests for back issues, and changes of address should be sent to Mathematical Sciences Publishers, Department of Mathematics, University of California, Berkeley, CA 94720-3840.

JoMMS peer-review and production is managed by EditFLow ${ }^{\circledR}$ from Mathematical Sciences Publishers.

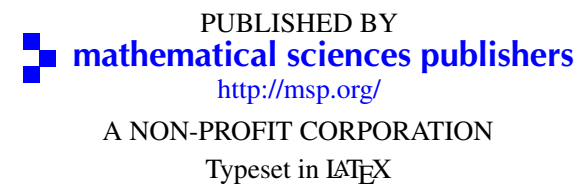

Copyright (C2011 by Mathematical Sciences Publishers 


\section{Journal of Mechanics of Materials and Structures}

\section{Volume 6, No. 7-8}

September-October 2011

\section{Special issue \\ Eleventh Pan-American Congress \\ of Applied Mechanics (PACAM XI)}

Preface

Adair R. Aguiar

949

Influence of specimen geometry on the Portevin-Le Châtelier effect due to dynamic strain aging

for the AA5083-H116 aluminum alloy

Rodrigo Nogueira de Codes and Ahmed Benallal

Dispersion relations for SH waves on a magnetoelectroelastic heterostructure with imperfect

interfaces

J. A. Otero, H. Calas, R. Rodríguez, J. Bravo, A. R. Aguiar and G. Monsivais

Numerical linear stability analysis of a thermocapillary-driven liquid bridge with magnetic stabilization

Yue Huang and Brent C. Houchens

Numerical investigation of director orientation and flow of nematic liquid crystals in a planar 1:4 expansion Pedro a. Cruz, Murilo F. Tomé, IAin W. Stewart and Sean McKee

Critical threshold and underlying dynamical phenomena in pedestrian-induced lateral vibrations of footbridges

Stefano LenCI and LAURA MARCHEgGiani

Free vibration of a simulation CANDU nuclear fuel bundle structure inside a tube

XUAN ZHANG and SHUdONG Yu

Nonlinear dynamics and sensitivity to imperfections in Augusti's model

D. Orlando, P. B. Gonçalves, G. Rega and S. LenCi

Active control of vortex-induced vibrations in offshore catenary risers: A nonlinear normal mode approach

CArlos E. N. MAZzilli and César T. SANCheS

Nonlinear electromechanical fields and localized polarization switching of piezoelectric macrofiber composites

Yasuhide Shindo, Fumio Narita, KoJi SATo and Tomo TAKeda

1089

Three-dimensional BEM analysis to assess delamination cracks between two transversely isotropic materials

Nicolás O. Larrosa, Jhonny E. Ortiz and Adrián P. Cisillino

Porcine dermis in uniaxial cyclic loading: Sample preparation, experimental results and modeling

A. E. Ehret, M. Hollenstein, E. MAzzA and M. Itskov

Analysis of nonstationary random processes using smooth decomposition

Rubens SAMpaio and Sergio Bellizzi

Perturbation stochastic finite element-based homogenization of polycrystalline materials

S. LePage, F. V. Stump, I. H. Kim and P. H. Geubelle

A collocation approach for spatial discretization of stochastic peridynamic modeling of fracture

Georgios I. Evangelatos and POL D. SPANOS 